\title{
Adjuvant Chemotherapy After Lobectomy for T1-2No Non-Small Cell Lung Cancer: Are the Guidelines Supported?
}

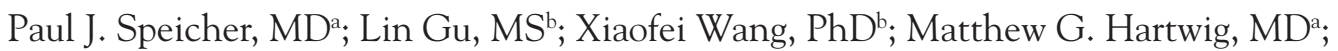

Thomas A. D'Amico, MDa ; and Mark F. Berry, MD

\begin{abstract}
Background: Evidence guiding adjuvant chemotherapy (AC) use after lobectomy for stage I non-small cell lung cancer (NSCLC) is limited. This study evaluated the impact of AC use and tumor size on outcomes using a large, nationwide cancer database. Methods: The effect of AC on long-term survival among patients who underwent lobectomy for margin-negative pathologic T1-2NOMO NSCLC in the National Cancer Data Base from 2003 to 2006 was estimated using the Kaplan-Meier method. The specific tumor size threshold at which AC began providing benefit was estimated with multivariable Cox proportional hazards modeling. Results: Overall 3,496 of 34,360 patients (10.2\%) who met inclusion criteria were treated with AC, although AC use increased over time from 2003, when only $2.7 \%$ of patients with tumors less than $4 \mathrm{~cm}$ and $6.2 \%$ of patients with tumors of $4 \mathrm{~cm}$ or larger received AC. In unadjusted survival analysis, AC was associated with a significant 5-year survival benefit for patients with tumors less than $4 \mathrm{~cm}(74.3 \%$ vs $66.9 \% ; P<.0001)$ and $4 \mathrm{~cm}$ or greater $(64.8 \%$ vs $49.8 \%$; $P<.0001)$. In subanalyses of patients grouped by strata of $0.5-\mathrm{cm}$ increments in tumor size, AC was associated with a survival advantage for tumor sizes ranging from 3.0 to $8.5 \mathrm{~cm}$. Conclusions: Use of AC among patients with stage I NSCLC has increased over time but remains uncommon. The results of this study support current treatment guidelines that recommend AC use after lobectomy for stage I NSCLC tumors larger than $4 \mathrm{~cm}$. These results also suggest that $A C$ use is associated with superior survival for patients with tumors ranging from 3.0 to 8.5 $\mathrm{cm}$ in diameter. (J Natl Compr Canc Netw 2015;13:755-761)
\end{abstract}

\section{Background}

Adjuvant chemotherapy (AC) after resection of stages II through IIIA non-small cell lung cancer (NSCLC) has repeatedly been shown to improve survival. ${ }^{1-6} \mathrm{NCCN}$ and ASCO therefore both recommend AC for patients with completely resected stage II or IIIA NSCLC. ${ }^{7,8}$ However, indications and benefits of $\mathrm{AC}$ for patients with earlier-stage NSCLC are less clear. Two large randomized trials failed to show a survival benefit associated with AC for early-stage, node-negative NSCLC, whereas a single-institution retrospective study of 119 patients

From the Departments of aSurgery and ${ }^{\mathrm{b} B i o s t a t i s t i c s}$ and Bioinformatics, Duke University Medical Center, Durham, North Carolina; and the 'Department of Cardiothoracic Surgery, Stanford University School of Medicine, Palo Alto, California.

Submitted October 12, 2014; accepted for publication

March 27, 2015.

The authors have disclosed that they have no financial interests, arrangements, affiliations, or commercial interests with the manufacturers of any products discussed in this article or their competitors.

This work was supported by the NIH-funded Cardiothoracic Surgery Trials Network (Drs. Hartwig and Berry), 5U01HL088953-05.

The data used in this study are derived from a deidentified National Cancer Data Base file. The American College of Surgeons and the who underwent lobectomy for stage IB NSCLC found that adjuvant platinum-based chemotherapy was associated with improved survival. ${ }^{3,6,9-11}$ The CALGB 9633 trial demonstrated an early survival advantage with $\mathrm{AC}$ for patients with T2NO NSCLC; however, this did not persist with longer follow-up. ${ }^{12}$ A subset analysis of this study did, however, reveal a survival advantage for tumors $4 \mathrm{~cm}$ or larger, and a similar pooled analysis of 2 clinical trials demonstrated a tumor size-chemotherapy effect. ${ }^{5,13}$

The current NCCN Clinical Practice Guidelines in Oncology (NCCN Guidelines) for NSCLC are based 
Speicher et al

on these data and do not recommend AC for patients with completely resected stage IB NSCLC, with the exception of individuals considered to be at high-risk for recurrence, including those with tumors $4 \mathrm{~cm}$ or larger (to view the most recent version of these guidelines, visit NCCN.org). ${ }^{7}$ However, use of these exploratory and unplanned subgroup analyses from the CALGB 9633 trial to direct patient care and influence guidelines regarding use of AC for completely resected node-negative NSCLC has been questioned. ${ }^{5,7,12}$ In light of current evidence and recommendations, most patients with earlystage NSCLC do not receive AC. ${ }^{14}$ The purpose of our study was to use a nationwide cancer database to provide the largest investigation to date evaluating the use of AC after lobectomy for T1-2NO NSCLC to better understand current practice patterns and evaluate the impact of tumor size on outcomes.

\section{Methods}

This retrospective analysis of patients with pathologic T1-2N0 NSCLC in the National Cancer Data Base (NCDB) was approved by the Duke University Institutional Review Board. The NCDB is a jointly administered effort by the American College of Surgeons Commission on Cancer $(\mathrm{CoC})$ and the American Cancer Society, and collects data from more than 1,500 CoC-approved US facilities. The NCDB is estimated to capture approximately $70 \%$ of all new cancer diagnoses annually, and currently contains more than 30 million patient records.

Patients diagnosed with pT1-2NOMO NSCLC from 2003 to 2006 were identified for inclusion based on International Classification of Diseases for Oncology, 3rd Edition (ICD-O-3) histology codes for NSCLC, and Facility Oncology Registry Data Standards (FORDS) procedure codes for lobectomy. This time period was chosen because patients diagnosed in 2007 and laterdonotcurrently havelong-termsurvival data available in the NCDB. Pathologic stage data were directly extracted based on AJCC 6th edition staging criteria. Tumor size data are recorded as the largest dimension of the diameter of the primary tumor, in millimeters. Patients who received induction therapy and those with missing data regarding the use of $\mathrm{AC}$ and/or tumor size were excluded from analysis. Only patients with negative margins after lobectomy were studied, because recommendations guiding the management of patients with marginpositive disease are notably different.

Baseline univariate comparisons of patient characteristics between the cohort of patients who received $\mathrm{AC}$ and the cohort of patients treated with surgery alone were made using Pearson chi-square test for discrete variables and Student $t$-test for continuous variables. Consistent with existing NCCN Guidelines, patients were then grouped according to whether their tumors were less than $4 \mathrm{~cm}$ or $4 \mathrm{~cm}$ or greater. Trends in the use of $\mathrm{AC}$ among the 2 size groups were assessed across the study time period. The effect of AC on long-term survival was estimated for each of the 2 groups. Patients were then grouped into strata by tumor size in increments of $1 \mathrm{~cm}$, and unadjusted median and 5-year survival rates were calculated by stratum. All unadjusted survival analyses were performed using the Kaplan-Meier product limit estimator. To identify the specific tumor size threshold where AC appeared to begin providing benefit, the study cohort was grouped by strata of increasing 5 $\mathrm{mm}$ in tumor size, and multivariable Cox proportional hazards models were developed within each strata, adjusting for patient age, sex, race, and Charlson/Deyo comorbidity score.

We made an affirmative decision to control for type I error at the level of all comparisons, and $P$ values less than .05 were considered statistically significant. Missing data were handled with complete case analysis in light of the substantial completeness of the NCDB data for the study population. All analyses were performed using SAS software (version 9.3; SAS Institute Inc., Cary, NC).

\section{Results}

A total of 34,360 patients underwent margin-negative lobectomy for pT1-2NOMO NSCLC between 2003 and 2006, of which 3,496 (10.2\%) were treated with AC, whereas the remaining 30,864 (89.8\%) were treated with surgery alone. Patients treated with $\mathrm{AC}$ were younger and had slightly lower Charlson comorbidity scores, but had significantly larger tumors and were more likely to have T2 disease (Table 1). Table 2 summarizes survival according to tumor size by $1 \mathrm{~cm}$ increments, and demonstrates progressively worse survival with increasing tumor size, ranging from a 75\% (95\% CI, 72\%-78\% ) 5-year survival rate among patients with tumors less than $1 \mathrm{~cm}$, to a rate of $46 \%(95 \% \mathrm{CI}, 44 \%$ - 
Adjuvant Chemotherapy for Early-Stage Lung Cancer

\begin{tabular}{|c|c|c|c|}
\hline & $\begin{array}{l}A C \\
(N=3,496)\end{array}$ & $\begin{array}{l}\text { No AC } \\
(\mathrm{N}=30,864)\end{array}$ & $P$ Value \\
\hline Patient age & & & $<.0001$ \\
\hline $\begin{array}{l}\text { Median } \\
\text { Q1, Q3 }\end{array}$ & $\begin{array}{l}63.0 \\
56.0,70.0\end{array}$ & $\begin{array}{c}69.0 \\
62.0,75.0\end{array}$ & \\
\hline Sex & & & .92 \\
\hline Male & $1,716(49.1 \%)$ & $15,121(49.0 \%)$ & \\
\hline Female & $1,780(50.9 \%)$ & $15,743(51.0 \%)$ & \\
\hline Race & & & .27 \\
\hline White & $3,143(89.9 \%)$ & $27,889(90.4 \%)$ & \\
\hline Black & $277(7.9 \%)$ & $2,237(7.2 \%)$ & \\
\hline Other & $76(2.2 \%)$ & $738(2.4 \%)$ & \\
\hline Charlson/Deyo score & & & $<.0001$ \\
\hline 0 & $1,991(57.0 \%)$ & $15,799(51.2 \%)$ & \\
\hline 1 & $1,169(33.4 \%)$ & $11,284(36.6 \%)$ & \\
\hline 2 & $336(9.6 \%)$ & $3,781(12.3 \%)$ & \\
\hline AJCC pathologic T stage & & & $<.0001$ \\
\hline 1 & $714(20.4 \%)$ & $18,436(59.7 \%)$ & \\
\hline 2 & $2,782(79.6 \%)$ & $12,428(40.3 \%)$ & \\
\hline Tumor size $(\mathrm{cm})$ & & & $<.0001$ \\
\hline Mean (SD) & $4.1(3.1)$ & $2.9(2.5)$ & \\
\hline Range & $(0.1-95.0)$ & (0.1-93.3) & \\
\hline Tumor size & & & $<.0001$ \\
\hline$<4 \mathrm{~cm}$ & $1,935(55.3 \%)$ & $24,610(79.7 \%)$ & \\
\hline$\geq 4 \mathrm{~cm}$ & $1,561(44.7 \%)$ & $6,254(20.3 \%)$ & \\
\hline
\end{tabular}

Abbreviation: AC, adjuvant chemotherapy.

49\%) for patients with tumors $7 \mathrm{~cm}$ or greater. Trends over time in the use of $\mathrm{AC}$, grouped according to the currently accepted size threshold of $4 \mathrm{~cm}$, are shown in Figure 1. Use of AC increased for both small $(<4 \mathrm{~cm})$ and large $(\geq 4 \mathrm{~cm})$ tumors, and although AC among the smaller tumors increased from $2.7 \%$ in 2003 to a peak of $9.7 \%$ in 2005 , use among patients with tumors $4 \mathrm{~cm}$ or greater increased from $6.2 \%$ in 2003 to a peak of $26.9 \%$ in 2005. In unadjusted survival analysis (Table 3 and Figure 2), AC was associated with a significant survival benefit both for patients with tumors less than $4 \mathrm{~cm}$ (5year survival, $74.3 \%$ vs $66.9 \% ; P<.0001)$ and $4 \mathrm{~cm}$ or larger (5-year survival, $64.8 \%$ vs $49.8 \%$; $P$ <.0001).

After grouping into cohorts by tumor size increments of $5 \mathrm{~mm}$ and subsequent multivariable Cox proportional hazards modeling, $\mathrm{AC}$ was associated with a statistically significant survival advantage for tumors starting at a threshold of $3.0 \mathrm{~cm}$ and ranging to an upper limit of $8.5 \mathrm{~cm}$. As shown in Figure 3, patients with small $(<3 \mathrm{~cm})$ tumors did not derive a significant adjusted survival benefit from AC, with a trend toward particularly bad outcomes for those with tumors less than $1 \mathrm{~cm}$ who received AC. Similarly, for tumors larger than $8.5 \mathrm{~cm}$, AC did not con- fer a statistically significant survival advantage after multivariable adjustment.

\section{Discussion}

In this NCDB study of more than 34,000 patients treated with lobectomy for pathologic T1-2N0M0 NSCLC in the United States from 2003 to 2006, we found that approximately $10 \%$ of patients were treated postoperatively with AC. Patients who were given $\mathrm{AC}$ were younger with fewer comorbidities, but with larger tumors. Survival among patients treated with AC for tumors ranging from 3.0 to 8.5 $\mathrm{cm}$ was significantly better than when $\mathrm{AC}$ was not given (Figure 3 ). The use of $\mathrm{AC}$ for both smaller and larger tumors seemed to increase from 2003 to 2005, corresponding to the time at which data from the CALGB 9633 study started becoming available and began shaping clinical practice.

Our results are consistent with the subgroup analysis from the prospective randomized CALGB 9633 trial for node-negative NSCLC tumors larger than $4 \mathrm{~cm},{ }^{2}$ and are generally in agreement with current NCCN Guidelines for NSCLC. ${ }^{7}$ Considering 
Speicher et al

\section{Table 2 Survival Estimates by Tumor Size}

\begin{tabular}{|lll|}
$\begin{array}{l}\text { Tumor Size } \\
(\mathbf{c m})\end{array}$ & $\begin{array}{l}\text { 5-Year Survival, \% } \\
(95 \% \mathrm{Cl})\end{array}$ & $\begin{array}{l}\text { Median Survival, y } \\
(95 \% \mathrm{Cl})\end{array}$ \\
\hline$<1.0$ & $75(72,78)$ & $8.8(8.6, \mathrm{NA})$ \\
$1.0-1.9$ & $73(72,74)$ & $8.6(8.4,8.9)$ \\
$2.0-2.9$ & $65(64,66)$ & $7.4(7.2,7.5)$ \\
$3.0-3.9$ & $61(60,62)$ & $6.5(6.4,6.8)$ \\
$4.0-4.9$ & $56(54,57)$ & $6.0(5.7,6.4)$ \\
$5.0-5.9$ & $53(50,55)$ & $5.6(5.1,6.2)$ \\
$6.0-6.9$ & $52(49,55)$ & $5.2(4.8,5.9)$ \\
$\geq 7$ & $46(44,49)$ & $4.4(3.9,4.8)$ \\
\hline
\end{tabular}

Abbreviation: NA, not achieved.

some of the concerns for basing clinical care solely on that unplanned subgroup analysis, the current study provides additional evidence to guide treatment and influence guidelines for this clinical situation. However, it should be noted that several other randomized trials have been unable to demonstrate any long-term survival benefit of $\mathrm{AC}$ after resection of stage I NSCLC. The International Adjuvant Lung Cancer Trial (IALT) included 1867 patients with surgically resected lung cancer who were randomly assigned to either cisplatin-based AC or observation, and reported a statistically significant survival benefit with cisplatin-based $\mathrm{AC}$ in patients with completely resected stage I, II, or III NSCLC. ${ }^{2}$ After 7.5 years of follow-up, however, more deaths were seen in the AC group, and the benefit of AC continued to decrease over time. ${ }^{15}$ Similar to the CALGB 9633 trial, the JBR.10 and ANITA trials both compared the effectiveness of adjuvant vinorelbine plus cisplatin versus observation in early-stage NSCLC, and showed benefit for patients with stages II and IIIA NSCLC, but not for those with stage IB disease after long-term follow-up., 3,69 Therefore, although the results of this current study provide a meaningful contribution to the existing literature addressing this unresolved question, they must be interpreted with caution.

Nevertheless, our study also demonstrates that long-term survival for patients treated with current optimal treatment (lobectomy) for early-stage NSCLC remains disappointingly low nationally, with 5-year survival rates of only $75 \%$ (95\% CI, $72 \%-78 \%$ ) after lobectomy for even the earliest stage I NSCLC tumors measuring less than $1 \mathrm{~cm}$. These survival data suggest that continued investigation

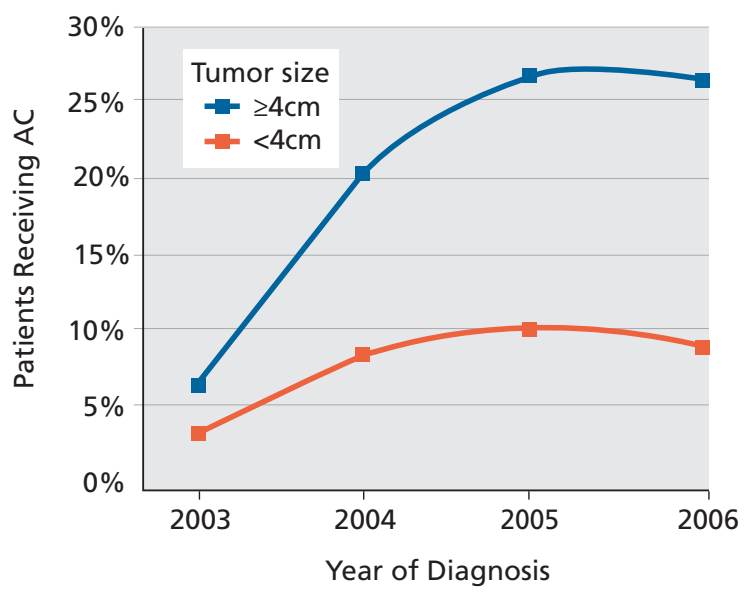

Figure 1 Trends in use of adjuvant chemotherapy (AC) by tumor size during the study period (2003-2006).

is necessary to improve outcomes for patients diagnosed with what should be the most curable instance of NSCLC, because surgery alone may not be adequate in up to a quarter of cases. Chemotherapy now has a category $2 \mathrm{~A}$ recommendation in the NCCN Guidelines for NSCLC as adjuvant treatment for patients with high-risk features ${ }^{7}$ including not only tumors larger than $4 \mathrm{~cm}$ but also poorly differentiated tumors, vascular invasion, wedge resection, visceral pleural involvement, and incomplete lymph node sampling $(\mathrm{Nx})^{5,10}$ Interestingly, multivariate survival analysis in this current study also shows that $\mathrm{AC}$ is significantly associated with improved survival for tumors 3.0 to $3.9 \mathrm{~cm}$, and supports the consideration of $\mathrm{AC}$ for smaller tumors in some situations. However, analysis in our study also found that the association of AC with improved survival was no longer statistically significant for tumors larger than 8.5 $\mathrm{cm}$ (Figure 3). Although this may simply represent a lack of statistical power because of a relative rarity of such large tumors, there may also be an underlying biological mechanism related to tumors that grow to very large sizes. Therefore, the risk of treatment failure with current strategies seems to be much more significant as tumors exceed $8 \mathrm{~cm}$, and future trials that develop and study new treatment regimens specifically for very large tumors should be considered.

Although this study showed that the use of AC for larger tumors increased from $6.2 \%$ in 2003 to approximately $27 \%$ in 2005 and 2006, clearly AC was still used in a minority of patients. This finding is consistent with those of other studies that have in- 
Adjuvant Chemotherapy for Early-Stage Lung Cancer

Table 3 Survival Estimates by Tumor Size and Use of AC

\begin{tabular}{|c|c|c|c|c|c|}
\hline Tumor Size & Treatment & $\begin{array}{l}\text { Median Survival, y } \\
(95 \% \mathrm{Cl})\end{array}$ & $\begin{array}{l}\text { 5-Year Survival, \% } \\
(95 \% \mathrm{Cl})\end{array}$ & $\begin{array}{l}\text { Hazard Ratio, AC } \\
\text { vs No AC }(95 \% \text { Cl) }\end{array}$ & $P$ Value \\
\hline \multirow[t]{2}{*}{$<4 \mathrm{~cm}$} & $A C$ & $8.7(8.4, N A)$ & $74.3(72.3,76.3)$ & $0.75(0.70,0.81)$ & $<.0001$ \\
\hline & No $A C$ & $7.6(7.5,7.7)$ & $66.9(66.3,67.5)$ & & \\
\hline \multirow[t]{2}{*}{$\geq 4 \mathrm{~cm}$} & $A C$ & $8(7.5, N A)$ & $64.8(62.3,67.2)$ & $0.6(0.55,0.65)$ & $<.0001$ \\
\hline & No $A C$ & $5(4.7,5.1)$ & $49.8(48.5,51.0)$ & & \\
\hline
\end{tabular}

Abbreviations: AC, adjuvant chemotherapy; NA, not achieved.

vestigated the use of $\mathrm{AC}$ for NSCLC. Although several recent randomized studies and meta-analyses have demonstrated a survival benefit for AC, guideline adherence after resection has been shown to be only $61 \% .{ }^{16}$ Barriers to the use of AC in nontrial settings likely include opinions of both physicians and patients regarding ability to tolerate chemotherapy and whether the potential benefits of $\mathrm{AC}$ outweigh the risks, particularly considering that the benefits are generally modest. ${ }^{4}$ Counseling patients on the potential benefits for their specific situation can be somewhat difficult, because the phase III studies included a heterogeneous group of stages. ${ }^{2,3,6}$ Previous studies have shown that as many as $26 \%$ of patients decline further treatment after surgery. ${ }^{14}$ It is likely that some patients did not feel the risks of $\mathrm{AC}$ were worth the potentially increased chance of long-term survival. The current study provides more accurate estimates of the potential benefits of $\mathrm{AC}$ use for patients who have undergone resection of early-stage, node-negative NSCLC in a nontrial setting, which can improve the shared decision-making process and better inform treatment recommendations.

In this study, 900 patients aged 70 years or older were given AC. Although there are no specific guidelines for the treatment of NSCLC based on age, counseling elderly patients on the potential benefits of AC may be more difficult than counseling younger patients, because most randomized studies either excluded older participants or enrolled very limited numbers. ${ }^{2,3,6,17,18}$ Even though NSCLC is generally a disease of the elderly, with a median age at diagnosis of 70 years, only $9 \%$ of patients in the meta-analysis of randomized trials were older than 70 years. ${ }^{19} \mathrm{Ad}$ ditionally, those older than 70 years were shown to have had a survival benefit from AC similar to that of younger patients, despite receiving lower doses and fewer cycles, having lower performance status, and having more non-lung cancer-related causes of death. ${ }^{18}$ Other retrospective analyses of both randomized study data and registry data have also demonstrated benefits of AC use among patients older than 65 years. ${ }^{20,21}$ In our study, AC was an independent predictor of improved survival even when age was considered.

The NCDB does also have some inherent limitations associated with the availability of clinical information related to the decision on whether to administer AC after surgery. Even though multivariable adjusted analysis can correct for measured covariates, the NCDB does not contain important
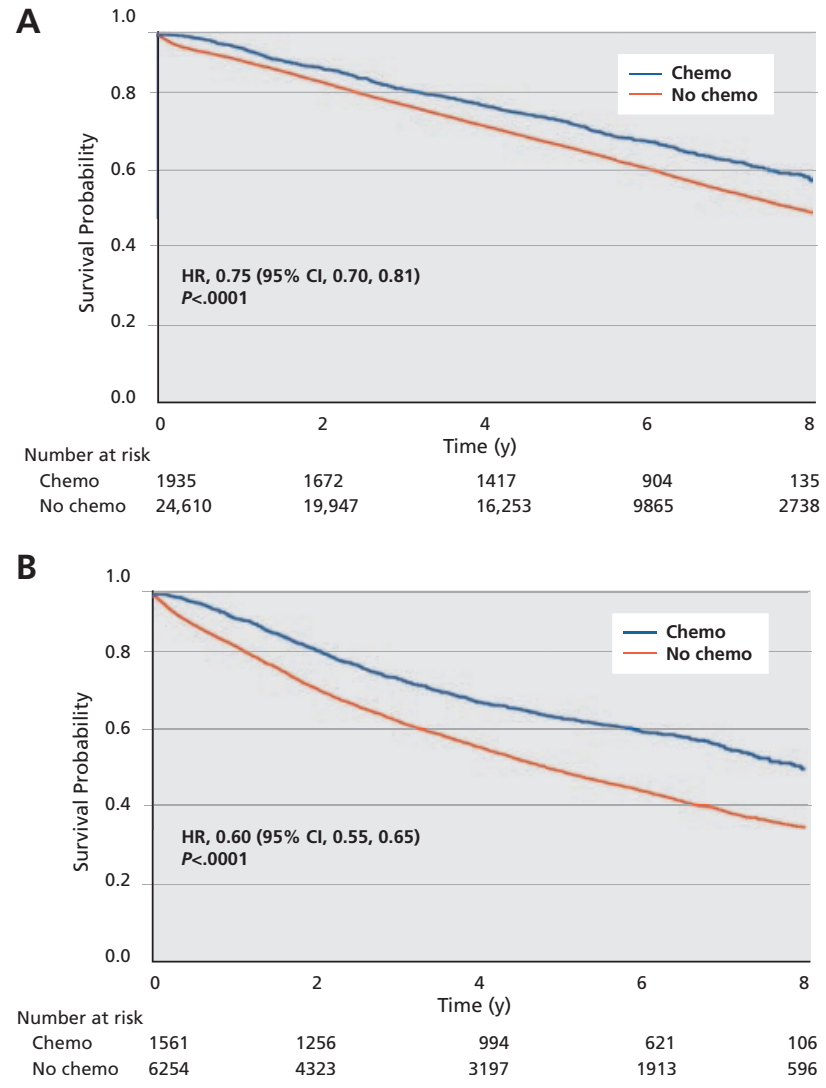

Figure 2 Kaplan-Meier survival curves for tumors (A) less than $4 \mathrm{~cm}$ and (B) $4 \mathrm{~cm}$ or greater.

Abbreviations: chemo, chemotherapy; HR, hazard ratio. 


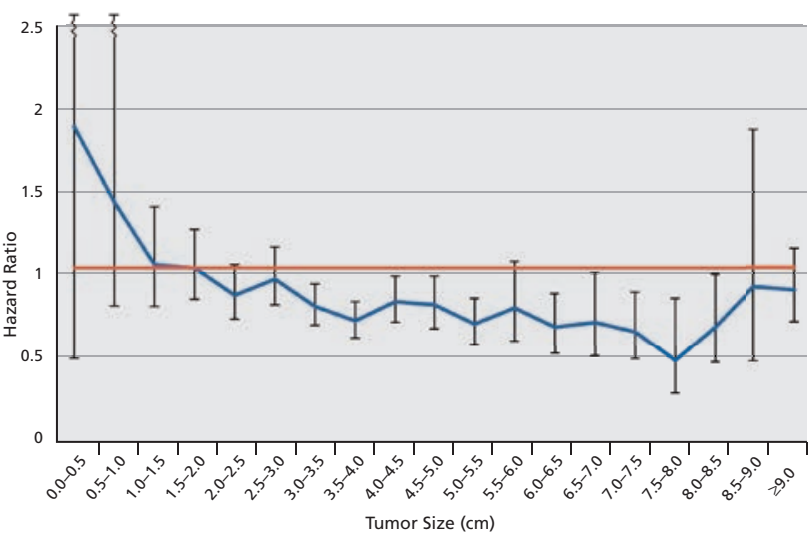

Figure 3 Plot of adjusted hazard of death associated with adjuvant chemotherapy after lobectomy for T1-2N0 grouped into strata by tumor size. The hazard ratios were determined from multivariable survival models performed on subsets of patients created by grouping the entire cohort by strata of tumor size in $0.5-\mathrm{cm}$ increments.

clinical variables, such as overall performance status, pulmonary function, specific comorbidities, and smoking status. AC may have been preferentially selected for patients who did well after surgery or who had better functional status, better pulmonary function, and less significant current and past smoking use, which are all factors that can impact treatment selection and outcomes, such as survival. This study has other limitations because of its retrospective nature and reliance on an administrative database in which some data may be missing. Additionally, we were unable to investigate disease-specific survival among our patient cohorts, because this was not currently available in the NCDB.

Use of the NCDB does provide the significant strength of allowing the investigation of a specific cancer substage with high power because of its population-based nature. Any prospective study would be very unlikely to accrue similar numbers of patients as analyzed in this study. In addition, randomized trials generally enroll selected participants with good functional status and a low number of comorbidities, and either exclude or tend to have a limited number of older participants. ${ }^{2,3,6,7,18}$ The study of patients in a nontrial setting using the NCDB may improve the generalizability of the findings and provide more appropriate expectations on which to base treatment guidelines. The results of our study support current treatment guidelines regarding $\mathrm{AC}$ use after lobectomy for stage I NSCLC. Surgeons and medical oncologists should carefully consider the use of AC after lobectomy for node-negative NSCLC tumors larger than $3 \mathrm{~cm}$, and particularly when tumors are larger than $4 \mathrm{~cm}$. However considering the promising but not definitively proven benefit of $\mathrm{AC}$ in this setting, the thoracic oncology community should focus on studies that investigate this topic to continue to try to improve outcomes after treatment for early-stage NSCLC.

\section{References}

1. Arriagada R, Auperin A, Burdett S, et al. Adjuvant chemotherapy, with or without postoperative radiotherapy, in operable non-small-cell lung cancer: two meta-analyses of individual patient data. Lancet 2010;375:1267-1277.

2. Arriagada R, Bergman B, Dunant A, et al. Cisplatin-based adjuvant chemotherapy in patients with completely resected non-small-cell lung cancer. N Engl J Med 2004;350:351-360.

3. Douillard JY, Rosell R, De Lena M, et al. Adjuvant vinorelbine plus cisplatin versus observation in patients with completely resected stage IB-IIIA non-small-cell lung cancer (Adjuvant Navelbine International Trialist Association [ANITA]): a randomised controlled trial. Lancet Oncology 2006;7:719-727.

4. Pignon JP, Tribodet H, Scagliotti GV, et al. Lung adjuvant cisplatin evaluation: a pooled analysis by the LACE Collaborative Group. J Clin Oncol 2008;26:3552-3559.

5. Strauss GM, Herndon JE, Maddaus MA, et al. Adjuvant paclitaxel plus carboplatin compared with observation in stage IB non-small-cell lung cancer: CALGB 9633 with the Cancer and Leukemia Group B, Radiation Therapy Oncology Group, and North Central Cancer Treatment Group Study Groups. J Clin Oncol 2008;26:5043-5051.

6. Winton $\mathrm{T}$, Livingston $\mathrm{R}$, Johnson $\mathrm{D}$, et al. Vinorelbine plus cisplatin vs. observation in resected non-small-cell lung cancer. $N$ Engl J Med 2005;352:2589-2597.

7. Ettinger DS, Wood DE, Akerley W, et al. NCCN Clinical Practice Guidelines in Oncology: Non-Small Cell Lung Cancer. Version 6, 2015 Available at: NCCN.org. Accessed May 7, 2015.

8. Pisters KM, Evans WK, Azzoli CG, et al. Cancer Care Ontario and American Society of Clinical Oncology adjuvant chemotherapy and adjuvant radiation therapy for stages I-IIIA resectable non small-cell lung cancer guideline. J Clin Oncol 2007;25:5506-5518.

9. Butts CA, Ding K, Seymour L, et al. Randomized phase III trial of vinorelbine plus cisplatin compared with observation in completely resected stage IB and II non-small-cell lung cancer: updated survival analysis of JBR-10. J Clin Oncol 2010;28:29-34.

10. Park SY, Lee JG, Kim J, et al. Efficacy of platinum-based adjuvant chemotherapy in T2aN0 stage IB non-small cell lung cancer. J Cardiothorac Surg 2013;8:151.

11. Roselli M, Mariotti S, Ferroni $P$, et al. Postsurgical chemotherapy in stage IB nonsmall cell lung cancer: Long-term survival in a randomized study. Int J Cancer 2006;119:955-960.

12. Katz A, Saad ED. CALGB 9633: an underpowered trial with a methodologically questionable conclusion. J Clin Oncol 2009;27:23002301; author reply 2301-2302.

13. Cuffe S, Bourredjem A, Graziano S, et al. A pooled exploratory analysis of the effect of tumor size and KRAS mutations on survival benefit from adjuvant platinum-based chemotherapy in node-negative non-small cell lung cancer. J Thorac Oncol 2012;7:963-972.

14. Zornosa C, Mamet R, Reid M, et al. Utilization of adjuvant therapy among completely resected non-small cell lung cancer (NSCLC) patients in the National Comprehensive Cancer Network (NCCN) Outcomes Database Project. J Clin Oncol 2010;28:7s.

15. Arriagada R, Dunant A, Pignon JP, et al. Long-term results of the International Adjuvant Lung Cancer Trial evaluating adjuvant cisplatinbased chemotherapy in resected lung cancer. J Clin Oncol 2010;28:35-42.

16. Wang Z, Askamit I, Tuscher L, et al. Rates of guideline adherence among US community oncologists treating NSCLC. Am J Manag Care 2013;19:185-192.

17. Scagliotti GV, Fossati R, Torri V, et al. Randomized study of adjuvant chemotherapy for completely resected stage I, II, or IIIA non-small-cell Lung cancer. J Natl Cancer Inst 2003;95:1453-1461. 
Adjuvant Chemotherapy for Early-Stage Lung Cancer

18. Waller D, Peake MD, Stephens RJ, et al. Chemotherapy for patients with non-small cell lung cancer: the surgical setting of the Big Lung Trial. Eur J Cardiothorac Surg 2004;26:173-182.

19. Früh M, Rolland E, Pignon JP, et al. Pooled analysis of the effect of age on adjuvant cisplatin-based chemotherapy for completely resected non-smallcell lung cancer. J Clin Oncol 2008;26:3573-3581.
20. Pepe C, Hasan B, Winton TL, et al. Adjuvant vinorelbine and cisplatin in elderly patients: National Cancer Institute of Canada and Intergroup Study JBR.10. J Clin Oncol 2007;25:1553-1561.

21. Wisnivesky JP, Smith CB, Packer S, et al. Survival and risk of adverse events in older patients receiving postoperative adjuvant chemotherapy for resected stages II-IIIA lung cancer: observational cohort study. BMJ 2011;343:d4013. 